\title{
Development of a Compact Cable-Driven Laparoscopic Endoscope Manipulator
}

\author{
Peter J. Berkelman, Philippe Cinquin, Jocelyne Troccaz, \\ Jean-Marc Ayoubi, and Christian Létoublon \\ Laboratoire TIMC-IMAG, Groupe GMCAO \\ Faculté de Médecine de Grenoble \\ Joseph Fourier University and CNRS 38706 La Tronche, France*
}

\begin{abstract}
This report describes continuing development of a novel compact surgical assistant robot to control the orientation and insertion depth of a laparoscopic endoscope during abdomenal surgery. The sterilizable manipulator is sufficiently small and lightweight that it can be placed directly on the body of the patient without interfering with other handheld instruments during abdomenal surgery. It consists of a round base, a clamp to hold the endoscope trocar, and two joints which enable azimuth rotation and inclination of the endoscope pivoting about the insertion point. The robot is actuated by thin cables inside flexible sleeves which are pulled by electric motors with a rack-and-pinion drive. Operation of the robot is demonstrated using an abdomenal surgical simulation trainer and experimental results are given.
\end{abstract}

\section{Introduction}

We have developed a compact and lightweight endoscope manipulator to serve as a surgical assistant during laparascopic surgery. In typical minimally invasive laparoscopic surgical procedures, the principal surgeon requires an assistant to hold the endoscope and an attached video camera so that the abdomenal cavity can be viewed by the surgeon while manipulating laparoscopic instruments with both hands through keyhole incisions. To avoid occupying a second surgeon, the human assistant can be replaced by a robotic endoscope manipulator. It is a simple task for a robotic assistant to move an endoscope to a desired orientation and insertion depth inside the abdomen, as the insertion point of the endoscope at its keyhole incision is fixed so that only three degrees of freedom are required. Furthermore, speed and accuracy requirements are low, and sensitive force control is unnecessary as the endoscope does not contact internal tissues.

Several robot systems have previously been developed for laparoscopic endoscope manipulation during surgery [1-4]. The AESOP[5,6] from Computer Motion and EndoAssist[7] from Armstrong Healthcare are two examples of commercially available endoscope manipulator devices. In addition, minimally invasive complete telerobotic surgical systems include the Da Vinci[8] system from

\footnotetext{
* This work is supported under RNTL grants (Ministère de L'Industrie, ANVAR, MMM Project) under the leadership of PRAXIM Inc.
} 


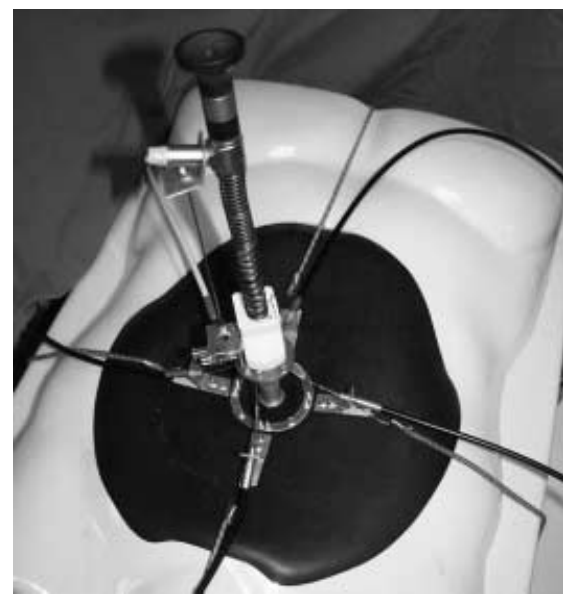

Fig. 1. Initial Endoscope Manipulator

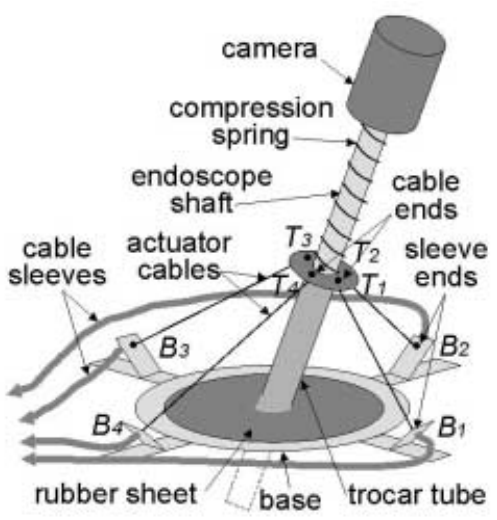

Fig. 2. Initial Endoscope Manipulator Schematic

Intuitive Surgical Systems and Zeus from Computer Motion. Clinical studies [9, $10,7]$ with various endoscope manipulator devices have indicated that the use of robotic endoscope assistants leads to a more stable image with no detrimental impact on efficacy or safety and a shorter duration of specific surgical interventions in many cases when compared to using a human assistant to hold the endoscope.

Although current endoscope manipulators perform well, they are generally large, heavy, complex, and expensive. In the limited space of a typical crowded operating room, the base of a conventional robot occupies a considerable amount of floor space next to the patient and the robot arm holding the endoscope may restrict full access to the abdomen of the patient. Hence it would be advantageous to have a much more lightweight, simple, and unobtrusive endoscope manipulator which would be easier to setup and use in conventional operating rooms. The novel features of our endoscope manipulator are that it is sufficiently lightweight that it can be strapped directly on the abdomen of the patient and that it is driven by cables inside flexible sleeves connected to motors in a separate module. The size and mass of our endoscope manipulator are nearly equivalent to that of the endoscope and camera.

Our first endoscope manipulator prototype used four cables connected directly between a circular base and the endoscope shaft to control endoscope orientation and a fifth cable along the endoscope shaft with compression springs to control the insertion depth. This system is described in detail in [11] and is shown in Figures 1 and 2. McKibben artificial pneumatic muscles $[12,13]$ attached to each cable were used for actuation.

The use of tensioned cables and sleeves to position medical instruments on a patient was proposed by Cinquin and Troccaz [14]. A cable-driven teleechography slave robot [15] uses the same principles to manipulate an ultrasound probe on a patient. 


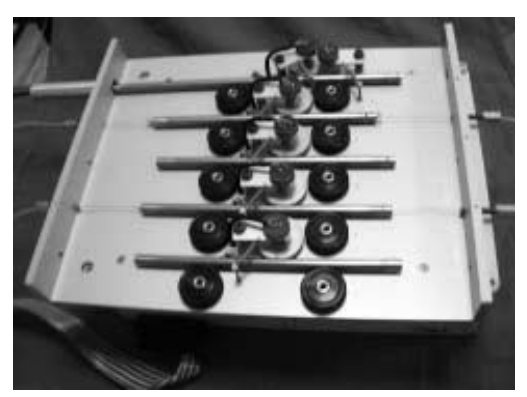

Fig. 3. Actuator Motors and Drives

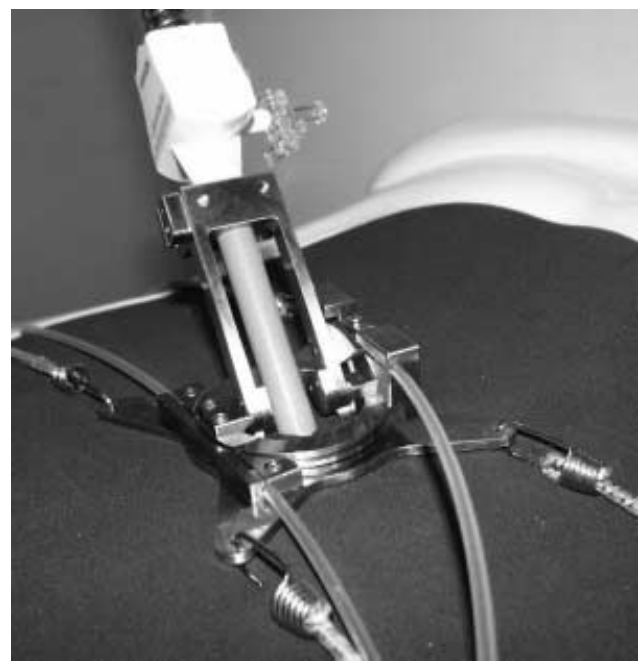

Fig. 4. New Laparoscopic Endoscope Manipulator on Simulation Abdomen

\section{Current Prototype}

Our current endoscope manipulator prototype is of the same overall shape and size as the previous prototype, but we use motorized rack-and-pinion drives in place of the pneumatic muscle actuators and a small mechanism with two rotational joints driven by pairs of antagonistic cables in place of the arrangement of four cables working together in parallel. The cable and spring configuration used to control the insertion depth of the endoscope is essentially the same as previously. Rotation of the endoscope on its axis is currently unactuated; another cable and motor could be added for this rotation if there is demand. The actuators are shown in Figure 3 and the new prototype mechanism is shown with the endoscope and trocar on a simulated abdomen in Figure 4.

Each rotational joint is driven by a single motor and rack with a cable attached at each end to rotate the joint in both directions. The endoscope insertion is actuated by a third motor and rack. Each motor is controlled in position by an analog feedback servocontrol circuit. The new motorized actuation system is more compact, more easily controlled, and more reliable than the previous pneumatic muscle actuators. The cables are replaceable and the tension is easily adjustable to compensate for stretching from repeated use.

In our new mechanism, the endoscope trocar is rigidly clamped to a small arm which is attached to the manipulator base through two rotational joints, instead of being supported by four tensioned cables and a flexible rubber sheet as with the previous prototype. The axes of the two rotation joints intersect at the endoscope insertion point incision. The endoscope insertion actuation cable can still be released easily at any time to remove the endoscope from the trocar for replacement or cleaning of the lens. 
The new endoscope orientation mechanism is more precise and more robust since there are no flexible components in the mechanism other than the actuation cables which drive the two rotational joints. Furthermore there are no vertical forces on the endoscope at its insertion point. As the two cables which drive each rotation joint are in direct opposition and the sum of their lengths remains constant throughout the motion of the joint, the ends of the cables can be attached to opposite ends of a single rack and motor, so that only one motor is necessary for each degree of freedom of the manipulator. For the same reasons, the kinematics and control of the new device is greatly simplified as well.

The mechanism is fabricated from stainless steel to be durable and easily sterilizable. The actuation cables are Teflon-coated and enclosed by Teflon sleeves. The cables and sleeves are disposable and would be replaced before each surgical procedure for sterility.

The relevant measured parameters of our current endoscope manipulator prototype are as follows:

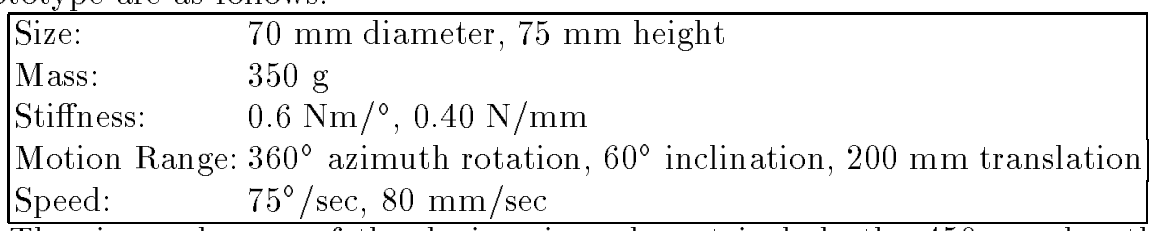

The size and mass of the device given do not include the $450 \mathrm{~mm}$ length and $340 \mathrm{~g}$ mass of the endoscope and camera. The limited stiffness in rotation is due to the elasticity of the actuator cables and in translation is due to the compression springs opposing the cable on the endoscope shaft.

\section{$3 \quad$ Kinematics}

The two axes of rotation of the manipulator mechanism intersect at the insertion point of the endoscope. The kinematics of the current manipulator are simple and straightforward, as the actuator cable lengths directly determine the position of the endoscope camera expressed in spherical coordinates $(\theta, \phi, \rho)$ :

$$
\begin{aligned}
\theta & =l_{1} / R_{1} \\
\phi & =l_{2} / R_{2} \\
\rho & =b-l_{3}
\end{aligned}
$$

where $l_{1}, l_{2}$, and $l_{3}$ are the positions of the three cable actuators, $R_{1}$ and $R_{2}$ are the radii of the horizontal base ring and vertical semicircle which are rotated by the cables, and $b$ is the length of the endoscope. The relations between the endoscope configuration expressed in Cartesian and in spherical coordinates is:

$$
\begin{aligned}
& x=\rho \cos \theta \sin \phi, \\
& y=\rho \sin \theta \sin \phi, \\
& z=\rho \cos \phi,
\end{aligned}
$$


and are inversible as:

$$
\begin{aligned}
& \rho=\sqrt{x^{2}+y^{2}+z^{2}} \\
& \theta=\tan ^{-1}(y / x) \\
& \phi=\cos ^{-1}\left(\frac{z}{\sqrt{x^{2}+y^{2}+z^{2}}}\right) .
\end{aligned}
$$

The vertical configuration of the endoscope corresponds to a kinematic singularity as the value of $\theta$ is undefined for $\phi=0$. The two coordinate systems are shown with a schematic of the endoscope in Figure 5.

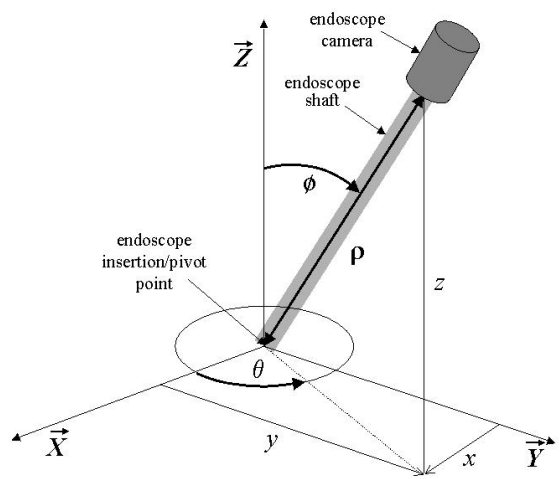

Fig. 5. Endoscope Manipulator Kinematics in Spherical and Cartesian Coordinates

If desired, noncontact 3D magnetic or optical localization systems could be used with the endoscope manipulator to provide absolute position feedback rather than the motor position feedback currently used. The localizer 3D coordinates would have to be registered to the endoscope pivot point to convert the localizer Cartesian $(x, y, z)$ coordinates to the spherical/actuator spherical/actuator $(\theta, \phi, \rho)$ coordinates using Equation 3 .

\section{Control Results}

Each position variable was measured by the potentiometer on its actuator to provide the response data shown in Figures 6, 7, and 8. Each coordinate variable is shown plotted against time in seconds in response to two step commands in opposite directions.

The $\theta$ angle response is the most accurate because there is no gravity loading on this horizontal rotation joint. This $90^{\circ}$ rotation was executed with the endoscope halfway extended at a $45^{\circ}$ inclination. The $\phi$ rotation angle response is quicker in one direction than the other due to the gravity loading torque from the mass of the endoscope when it is not vertical. The translation response $\rho$ is 
visibly less precise because it is actuated by only one cable with restoring forces provided by the compression springs on the shaft of the endoscope, whereas two cables operate antagonistically to actuate each of the two rotational joints. The response performance of the manipulator as shown is sufficient for a surgical assistant robot, where desired speeds are low and an surgeon operates the robot directly.

\section{Current Work}

Preliminary testing of our new prototype has indicated the need for several minor modifications to be done to improve the performance and practicality of the endoscope manipulator: The lever arm which clamps to the trocar should be shortened so to take up less space, reduce weight, and allow the trocar to be placed in a better position. The trocar clamp should be releasable by hand, so it could be replaced easily during surgery if required. The actuator cables and sleeves and their connection to the endoscope manipulator mechanism must be reconfigured to interfere less with the surgeon's instruments by being attached to the mechanism together in the same location and direction. To reduce the maximum required cable tension, the size of the pulley on the endoscope inclination joint should be increased and and a spring may be added to counterbalance the weight of the endoscope camera.

The currently implemented operator interfaces are rudimentary; motion commands for the endoscope manipulator can be given only by computer mouse or by rotating potentiometer dials. We have begun to investigate various possibilities for more sophisticated hands-free control which would be suitable for use in surgery, such as eye tracking, head tracking, voice commands, or multifunctional pedals. We also plan to replace the current PC control system with an embedded controller to make the complete system easily portable in a briefcase-sized package. Once these modifications are completed we plan to proceed with clinical validation.

\section{Conclusion}

We have developed and demonstrated a working prototype of a laparascopic surgical assistant endoscope manipulator which is especially lightweight, compact, and unobtrusive due to cable-driven actuation and being attached directly to the abdomen of the patient. The current prototype is an improvement of our previous mechanism.

\section{Acknowledgements}

The work of Eric Boidard and the expertise of Gildas de Saint Albin have significantly contributed to this project. The new prototype was designed and fabricated in cooperation with Alpes Instruments Inc. of Meylan, France. 


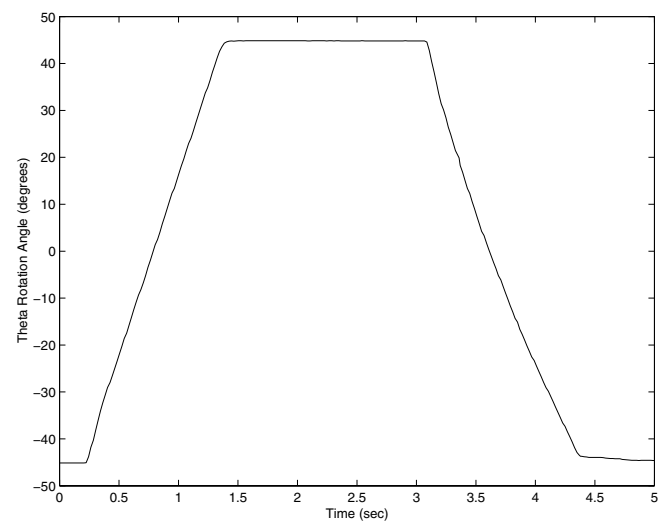

Fig. 6. Theta Response to Step Command Inputs

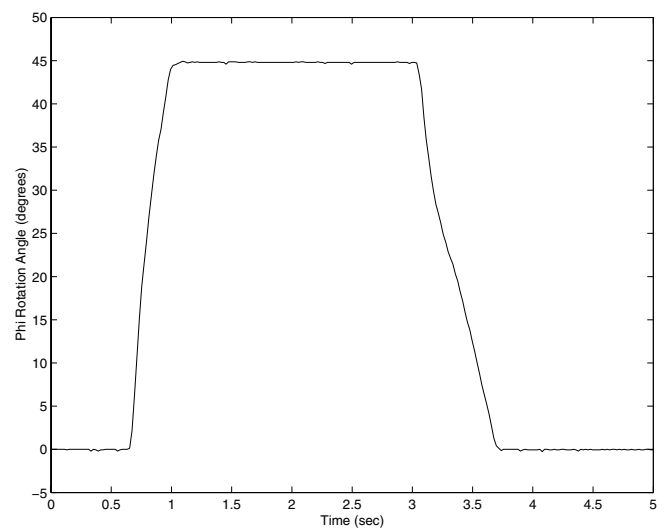

Fig. 7. Phi Response to Step Command Inputs

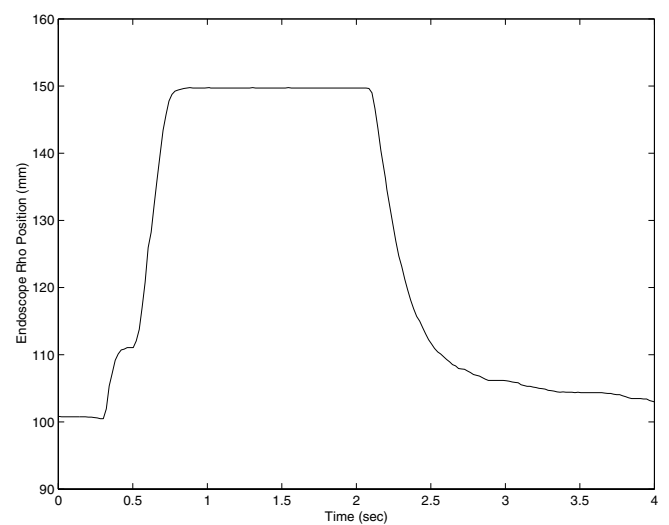

Fig. 8. Rho Response Step Command Inputs 


\section{References}

1. E. Kobayashi, K. Masamune, I. Sakuma, T. Dohi, and D. Hashimoto, "A new safe laparoscopic manipulator system with a five-bar linkage mechanism and optical zoom," Computer Aided Surgery, vol. 4, no. 4, pp. 182-192, 1999.

2. R. H. Taylor, J. Funda, B. Eldridge, S. Gomory, K. Gruben, D. LaRose, M. Talamini, L. Kavoussi, and J. A. Anderson, "Telerobotic assistant for laparoscopic surgery," in Computer Integrated Surgery: Technology and Clinical Applications (R. H. Taylor, S. Lavallee, G. C. Burdea, and R. Mosges, eds.), pp. 581-592, MIT Press, 1995.

3. V. F. Munoz et al., "Design and control of a robotic assistant for laparoscopic surgery," in 9th International Suymposium on Intelligent Robotic Systems, (Toulouse, France), July 2001.

4. G. F. Buess, A. Arezzo, M. O. Schurr, F. Ulmer, H. Fisher, L. Gumb, T. Testa, and C. Nobman, "A new remote-controlled endoscope positioning system for endoscopic solo surgery-the fips endoarm," Surgical Endoscopy, vol. 14, pp. 395-399, 2000.

5. W. P. Geis, H. C. Kim, E. J. B. Jr., P. C. McAfee, and Y. Wang, "Robotic arm enhancement to accommodate improved efficiency and decreased resource utilization in complex minimally invasive surgical procedures," in Medicine Meets Virtual Reality: Health Care in the Information Age, (San Diego), pp. 471-481, 1996.

6. J. M. Sackier and Y. Wang, "Robotically assisted laparoscopic surgery: from concept to development," in Computer Integrated Surgery: Technology and Clinical Applications, pp. 577-580, MIT Press, 1995.

7. S. Aiono, J. M. Gilbert, B. Soin, P. A. Finlay, and A. Gordon, "Controlled trial of the introduction of a robotic camera assistant (EndoAssist) for laparoscopic cholecystectomy," in 11th Annual Scientific Meeting Society for Minimally Invasive Therapy, (Boston), September 1999.

8. G. S. Guthart and J. K. Salisbury, "The Intuitive (TM) telesurgery system: Overview and application," in International Conference on Robotics and Automation, (San Francisco), pp. 618-621, IEEE, April 2000.

9. L. Mettler, M. Ibrahim, and W. Jonat, "One year of experience working with the aid of a robotic assistant (the voice-controlled optic holder AESOP) in gynaecological endoscopic surgery," Human Reproduction, vol. 13, pp. 2748-2750, 1998.

10. L. R. Kavoussi, R. G. Moore, J. B. Adams, and A. W. Partin, "Comparison of robotic versus human laparoscopic camera control," Journal of Urology, vol. 154, pp. 2134-2136, 1995.

11. P. J. Berkelman, P. Cinquin, J. Troccaz, J. Ayoubi, C. Letoublon, and F. Bouchard, "A compact, compliant laparoscopic endoscope manipulator," in International Conference on Robotics and Automation, (Washington D.C.), IEEE, May 2002.

12. V. L. Nickel, M. D. J. Perry, and A. L. Garrett, "Development of useful function in the severely paralyzed hand," Journal of Bone and Joint Surgery, vol. 45A, no. 5, pp. 933-952, 1963.

13. H. F. Schulte, "The characteristics of the McKibben artificial muscle," in The application of external power in prosthetics and orthotics, pp. 94-115, Washington D. C.: National academy of sciences-National research council, 1961.

14. P. Cinquin and J. Troccaz, "Système télécommandable de positionnement sur un patient d'un dispositif d'observation intervention," Patent 99 09363, France, 1999.

15. A. Vilchis-Gonzales, J. Troccaz, P. Cinquin, F. Courreges, G. Poisson, and B. Tondu, "Robotic tele-ultrasound system (TER): Slave robot control," in IFAC, 2001. 